\title{
Presence of multimeric isoforms of human C-reactive protein in tissues and blood
}

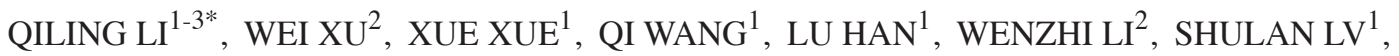 \\ DONG LIU ${ }^{1}$, JENDAI RICHARDS ${ }^{2}$, ZHUJUN SHEN $^{4}$, LI MA $^{2}$ and QING SONG ${ }^{1-3 *}$ \\ ${ }^{1}$ Department of Obstetrics and Gynecology, First Affiliated Hospital, Xi'an Jiaotong University, Xi'an, Shaanxi 710061, \\ P.R. China; ${ }^{2}$ Cardiovascular Research Institute, Morehouse School of Medicine, Atlanta, GA 30310, USA; \\ ${ }^{3}$ Centre of Big Data, First Affiliated Hospital, Xi'an Jiaotong University, Xi'an, Shaanxi 710061; \\ ${ }^{4}$ Department of Cardiology, Peking Union Medical College Hospital, Chinese Academy of Medical Sciences, \\ Peking Union Medical College, Beijing 100730, P.R. China
}

Received June 22, 2016; Accepted September 20, 2016

DOI: $10.3892 / \mathrm{mmr} .2016 .5922$

\begin{abstract}
The baseline concentration of C-reactive protein (CRP) has been associated with a wide array of human diseases. In epidemiological studies and in the clinic, CRP is typically measured as a pentamer, composed of 5 identical CRP subunits. The present study aimed to determine whether other isoforms were present in the blood by examining CRP conformations. Transgenic rats expressing human CRP under the mouse albumin promoter were generated and genotyped. Non-reducing western blotting was performed using the blood and tissues of transgenic rats and human patients. CRP concentrations in human blood were examined by enzyme-linked immunosorbent assay. In addition to the pentameric isoform, CRP was detected as a trimer and tetramer in the blood of human CRP transgenic rats. Furthermore, trimeric and tetrameric CRP was observed in various tissues, including aorta, liver, kidney, pancreas, heart and skeletal muscle. Notably, these two isoforms appeared to be age-associated, as they were detected only in the blood and tissues of older transgenic
\end{abstract}

Correspondence to: Professor Qing Song, Cardiovascular Research Institute, Morehouse School of Medicine, 720 Westview Drive SW, Atlanta, GA 30310, USA

E-mail: qsong@msm.edu

Professor Qiling Li, Department of Obstetrics and Gynecology, First Affiliated Hospital, Xi'an Jiaotong University, 277 West Road, Xi'an, Shaanxi 710061, P.R. China

E-mail: liqiling@mail.xjtu.edu.cn

*Contributed equally

Abbreviations: CRP, C-reactive protein; ELISA, enzyme-linked immunosorbent assay; hsCRP, high-sensitivity C-reactive protein; pCRP, pentameric C-reactive protein; mCRP, monomeric C-reactive protein

Key words: C-reactive protein, aging, epidemiology, cardiovascular intervention, cardiovascular disease rats. The existence of additional CRP isoforms was confirmed in the blood of human patients by non-reducing western blotting. Clinical and epidemiological studies typically focus on CRP concentration. However, the results of the present study suggest that, in addition to concentration, CRP conformation may require analysis.

\section{Introduction}

C-reactive protein (CRP) is an acute phase protein synthesized primarily by the liver that is involved in the systemic response to inflammation and is regulated by cytokines like interleukin (IL)-6, IL-1 $\beta$ and tumor necrosis factor- $\alpha(1,2)$. In clinical practice, it has been used as a non-specific marker of inflammation and atherosclerotic cardiovascular disease. It is unknown if CRP plays an active role as an etiologic factor in cardiovascular disease. Some studies show that the effect of CRP on atherogenesis may include interactions with other factors of immunity and inflammation, such as the complement system, as well as a direct effect of CRP on the cells involved in atherosclerotic lesions $(1,2)$. CRP has also shown value in predicting disease risk and assisting decision making on treatment for a series of diseases: Baseline CRP concentration has strong predictive and prognostic values for future cardiovascular events (3-5). The Centers for Disease Control and Prevention and the American Heart Association reported that it is reasonable to measure CRP as an adjunct to the measurement of established risk factors in order to assess the risk of coronary heart disease (5).

High-sensitivity CRP (hsCRP) typically refers to the pentameric form of CRP, which is a cyclic structure comprised of 5 identical 21.5-kDa subunits (6). Pentameric CRP (pCRP) has a recognition site that binds to phosphocholine and an effector site that binds to complement component $\mathrm{Clq}, \mathrm{Fc} \gamma$ receptors or other putative CRP receptors (7-9). In addition to pCRP, monomeric CRP (mCRP) has been detected in the intimal region of blood vessels in healthy human tissues and the atherosclerotic plaque, whereas pCRP was not observed in healthy or diseased arteries (10-12). The majority of previous studies have revealed the absence of pCRP and mCRP in healthy tissues, including 
blood vessels (12-14). Certain studies have indicated that pCRP and mCRP have opposing functions $(11,15-18)$.

The aim of the present study was to investigate the conformations of human CRP in humans and transgenic rats. The present study revealed, to the best of our knowledge for the first time, that human CRP may be naturally present in other multimeric isoforms, the trimer and tetramer. Notably, the appearance of these additional isoforms appears to be age-associated. The existence of different isoforms in different tissues may facilitate understanding of the functional impact of CRP in cardiovascular disease. In addition, when measuring $\mathrm{CRP}$ in the clinic, it may be useful to analyze the conformational structures.

\section{Materials and methods}

Ethics statement. All animal experiments were performed with the approval of the Animal Care Committee of the Universities of Morehouse School of Medicine (Atlanta, GA, USA), and conformed to the Guide for the Care and Use of Laboratory Animals produced by the National Institutes of Health (Bethesda, MD, USA). The present study meets the ARRIVE guidelines requirements for reporting (www.nc3rs. org.uk/arrive-guidelines). Animals were housed in the Center for Laboratory Animal Resources of Morehouse School of Medicine. Transgenic CRP rats were maintained under a 12-h light-dark cycle with ad libitum access to water and a standard rat chow diet (Laboratory Rodent Diet 5001; LabDiet, St. Louis, MO, USA). The animal room temperature was maintained at $22 \pm 3^{\circ} \mathrm{C}$, relative humidity was held at $30-70 \%$, and air was exchanged 15 times/h.

The informed consent procedure of human experiments applied at the First Affiliated Hospital of Xi'an Jiaotong University (Xi'an, China) was in accordance with the approved guidelines. The present study was approved by the Institutional Review Board of Xi'an Jiaotong University, and written informed consent was obtained from patients.

Generation and genotyping of albumin-human CRP $(A L B-h C R P)$ transgenic rats. A fragment containing 21 nucleotides upstream to the transcription initiation site, exons and the intron, and $1.2 \mathrm{~kb}$ of 3 '-flanking region of the human CRP gene, was amplified from human genomic DNA purchased from the Coriell Institute for Medical Research (Camden, NJ, USA) and cloned into a TA cloning vector (Invitrogen; Thermo Fisher Scientific, Inc., Waltham, MA, USA). This fragment was then released by restriction enzymes and inserted into a vector downstream of the mouse albumin enhancer/promoter element. The construct was verified by restriction enzyme mapping and DNA sequencing. The plasmid ALB-hCRP contained a $3.5-\mathrm{kb}$ mouse albumin enhancer element $(-8.5$ to $-12 \mathrm{~kb})$ and a $0.3 \mathrm{~kb}$ promoter element $(-300$ to +22$)$ as well as the human CRP gene. A 4.3-kb fragment (Fig. 1) was released from the ALB-hCRP construct by HindIII/BglI digestion, purified with agarose gel electrophoresis and a QIAquick Polymerase Chain Reaction (PCR) Purification kit (Qiagen, Inc., Valencia, CA, USA). To generate transgenic founders, fertilized eggs from Sprague Dawley (SD) rats were microinjected with the transgene fragment; this was performed by the University of Michigan Transgenic Animal Model Core
Facility (Ann Arbor, MI, USA). Transgenic founders were identified by genotyping with the primers presented in Fig. 1, and mated with non-transgenic rats to produce F1 progeny $(19,20)$. Rats were anesthetized with isoflurane (Laser Animal Health, Sydney, Australia) and a $0.2-\mathrm{cm}$ tail biopsy was excised and collected. Genomic DNA was extracted from the tail biopsies with DNeasy Blood \& Tissue Kit (catalog no. 69506; Qiagen $\mathrm{GmbH}$, Hilden, Germany) and PCR was performed in $20-\mu 1$ reactions, containing $5 \mathrm{ng}$ genomic DNA, $5 \mu \mathrm{M}$ each primer (forward, 5'-ACA TAC GCA AGG GAT TTA GTC-3'; reverse, 5'-AAC AGC TTC TCC ATG GTC AC-3'), 5 mM dNTP, $2 \mu 1$ 10X PCR buffer and 1 unit Taq polymerase (catalog no. 180384042; Thermo Fisher Scientific, Inc.). Amplification was performed for 35 cycles of $94^{\circ} \mathrm{C}$ for $30 \mathrm{sec}, 55^{\circ} \mathrm{C}$ for $30 \mathrm{sec}$ and $72^{\circ} \mathrm{C}$ for $1 \mathrm{~min}$, using a GeneAmp ${ }^{\circledR}$ PCR System 9600 (Applied Biosystems; Thermo Fisher Scientific, Inc.). PCR products were then visualized on $1 \%$ agarose gels following ethidium bromide staining.

Samples. Rats were anesthetized using isoflurane. Blood samples were collected from anesthetized transgenic rats and three human patients who volunteered for this research from the Inpatient Cardiology Department of the First Affiliated Hospital of Xi'an Jiaotong University (Table I) in 10-ml tubes without anticoagulant. Samples were allowed to clot for $1 \mathrm{~h}$ at room temperature, and then centrifuged at $2,220 \times \mathrm{g}$ at $4^{\circ} \mathrm{C}$ for $10 \mathrm{~min}$ to separate the serum. All 12 rats were sacrificed with $70 \% \mathrm{CO}_{2}$ following anesthesia at the same time at 9,14 and 37 weeks of age, as the 9- and 37-week-old rats belonged to separate generations. Liver, pancreas, kidney, aorta, heart and muscle were collected from transgenic rats. Harvested tissues (30 mg) were snap frozen in liquid nitrogen and homogenized with a Teflon glass homogenizer in extraction medium (protease inhibitor cocktail (catalog no. P8340-5; Sigma-Aldrich; Merck Millipore, Darmstadt, Germany) diluted 1:30 in tissue protein extraction reagent (catalog no. 78510, Thermo Fisher Scientific, Inc.). Following centrifugation at $13,200 \mathrm{x} g$ at $4^{\circ} \mathrm{C}$ for $10 \mathrm{~min}$, an aliquot of the supernatant was used to determine the protein concentrations using the bicinchoninic acid assay and a BioPhotometer (Eppendorf, Hamburg, Germany).

Enzyme-linked immunosorbent assay (ELISA) for human $C R P$. The concentration of human CRP in the samples was measured by ELISA using a commercial hsCRP ELISA kit (catalog no. 961CRP01H-96; Helica Biosystems, Inc., Santa Ana, CA, USA). Samples were measured in duplicate, and experiments were repeated more than three times.

Western blot analysis. Total proteins $(20 \mu \mathrm{g})$ were denatured at $70^{\circ} \mathrm{C}$ for $10 \mathrm{~min}$, mixed with NativePAGE Sample Buffer (Thermo Fisher Scientific, Inc.), electrophoresed on Novex 4-12\% Tris Glycine Midi Protein Gels (Thermo Fisher Scientific, Inc.) and transferred to polyvinylidene difluoride membranes. Membranes were blocked with 5\% milk for $1 \mathrm{~h}$ and incubated with anti-human CRP antibodies overnight: Mouse anti-CRP (1:500; clone, C6; catalog no. M86284M; Meridian Life Science, Inc., Cincinnati, OH, USA), mouse anti-CRP (1:500; clone, C5; catalog no. M86005M; Meridian Life Science, Inc.) and mouse anti-CRP (1:500; polyclonal; catalog no. ab52687; Abcam, Cambridge, MA, 


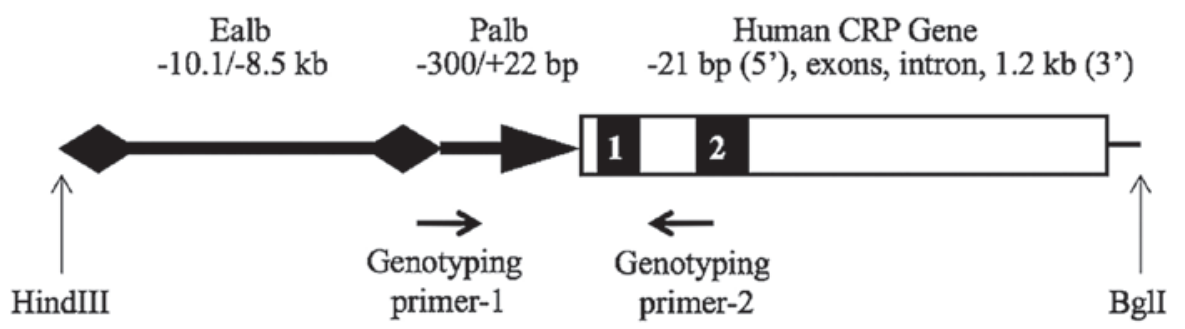

Figure 1. Albumin-human CRP transgene. The 4.3-kb transgene fragment for microinjection was composed of an enhancer (-10.1/-8.5 kb) and promoter $(-300 /+22 \mathrm{bp})$ of the mouse albumin gene, and a genomic fragment of the human CRP gene spanning from -21 bp upstream of the transcription initiation site to $1.2 \mathrm{~kb}$ downstream to exon 2. Genotyping primers are indicated by horizontal arrows. CRP, C-reactive protein; Ealb, albumin enhancer; Palb, albumin promoter.

USA). The membranes were washed 3 times with TBST (1\% serum albumin in $50 \mathrm{mM}$ Tris- $\mathrm{HCl}, \mathrm{pH} 7.4$, containing $0.05 \%$ Tween-20) at room temperature and incubated with a horseradish peroxidase-conjugated goat anti-mouse IgG secondary antibody (1:2,000; catalog no. sc-2005; Santa Cruz Biotechnology, Inc., Dallas, TX, USA) at room temperature for $1 \mathrm{~h}$. Membranes were washed three times, visualized with SuperSignal West Pico Chemilluminescent substrate (Thermo Fisher Scientific, Inc.) for $5 \mathrm{~min}$ and exposed to X-ray film. Densitometry was performed using Image J 2.1.4.7 software (National Institutes of Health).

Statistical analysis. Data are expressed as the mean \pm standard deviation. Differences between groups were analyzed using ANOVA for more than two variables. Where ANOVA revealed a significance, post hoc comparisons were made by means of Tukey's range test. Statistical analysis was performed using SAS 9.3 software; SAS Institute, Inc., Cary, NC, USA. $\mathrm{P}<0.05$ was considered to indicate a statistically significant difference.

\section{Results}

Characterization of transgenic rats. A total of nine founders were identified. Transgenic founders were bred with wild-type $\mathrm{SD}$ rats to establish transgenic lines. The offspring of these breeding pairs containing the ALB-hCRP transgene were identified by PCR using primers presented in Fig. 1 and genomic DNA obtained from tail biopsies. In addition, human hsCRP ELISA was performed on the founders to identify the transgenic lines with detectable levels of human CRP in the blood (Fig. 2). Lines were selected for use in subsequent experiments on the basis of this ELISA. Male heterozygous offspring were included in the present study.

Multimeric isoforms of human CRP in the blood of transgenic rats. Blood was collected from the tail veins of 11 human CRP transgenic rats from the 4 independent transgenic founder lines. In the non-reducing western blot analysis it was observed that CRP was detected as a series of bands, rather than as a pentamer only; in addition to bands the size of pCRP, three additional bands were observed, with sizes consistent with tetrameric, trimeric and dimeric CRP (Fig. 3A). The CRP band patterns were reproducible using different CRP-specific antibodies (Fig. 3B) and were observed to be identical between serum and plasma (Fig. 3C). mCRP was not observed in these experiments
(Fig. 3). Notably, the appearance of the trimer and tetramer appeared to be associated with age, as these non-traditional multimeric isoforms were observed only in the 14- and 37-week-old rats, and not in the 9-week-old rats (Fig. 3). The fractional composition of different CRP multimeric isoforms is presented for each age group (Fig. 3A and Table II). To examine the cross-reactivity of antibodies for human and rat CRP, a 38-week-old wild-type control was included (lane 12, Fig. 3A), in which only the pentamer and dimer were observed.

To assess the specificity of the CRP-specific antibodies used in the western blotting experiments, three antibodies were purchased from two companies and compared using serum and plasma from transgenic rats (Fig. 3). The results were identical with different antibodies. M86284M (Meridian Life Science, Inc.) and ab52687 (Abcam) cross-reacted with the rat endogenous CRP, which revealed only pentamer and dimer in the blood of the wild-type rat (Lane 12, Fig. 3A and B). The bands of tetramer and trimer were unique in transgenic rats compared with the wild-type rats, indicating that these two multimeric isoforms were specifically derived from transgenic rats. All three anti-human CRP antibodies used in western blotting consistently detected trimers and tetramers in the transgenic rats (Fig. 3). This multimeric phenomenon was observed in all three CRP transgenic lines (Fig. 3).

Multimeric isoforms of human CRP in various tissues of transgenic rats. In addition to serum and plasma, six tissues of the ALB-hCRP transgenic rats were analyzed for human CRP protein expression levels: Aorta, liver, kidney, pancreas, heart and skeletal muscle. mCRP was not observed in the tissues, as for the blood. Similar to the observations in blood, CRP was present in a series of bands in all tissues analyzed using non-reducing western blotting (Fig. 4A). Notably, the appearance of trimers and tetramers again appeared age-associated, as these non-traditional multimeric isoforms were observed only in the 37-week-old rats and not in the 9-week-old rats. It is possible that the inter-subunit disulfide bond may give rise to additional oligomeric bands (21). Furthermore, the additional bands may represent a homogenous protein complex formed by dissociated CRP subunits or a heterogenous protein complex formed by dissociated CRP subunits and other plasma or tissue proteins $(22,23)$.

Multimeric isoforms of CRP in the blood of human patients. Three human patients were recruited, among whom, individuals $\mathrm{a}$ and $\mathrm{b}$ were patients with coronary heart disease, 
Table I. The characteristics of human participants.

Patient

\begin{tabular}{lccc}
\cline { 2 - 4 } Characteristic & $\mathrm{a}$ & $\mathrm{b}$ & $\mathrm{c}$ \\
\hline Gender & Male & Male & Male \\
Age (year) & 65 & 60 & 62 \\
Diagnosis & Myocardial & Coronary & Congenital \\
& infarction & disease & heart disease \\
Levels of high-sensitivity C-reactive protein $(\mu \mathrm{g} / \mathrm{ml})$ & 32.2 & 25.8 & 0.0
\end{tabular}

Table II. Human C-reactive protein concentrations in the serum of transgenic rats at various ages, quantified from western blot analysis using the M86284M antibody (Meridian Life Sciences, Inc.) and analyzed using Image J software.

\begin{tabular}{|c|c|c|c|c|c|c|}
\hline \multirow[b]{2}{*}{ Isoform } & \multicolumn{3}{|c|}{ Mean \pm standard deviation } & \multicolumn{3}{|c|}{ P-value } \\
\hline & 9 week & 14 week & 37 week & 9 vs. 14 week & 9 vs. 37 week & 14 vs. 37 week \\
\hline Pentamer & $77.49 \pm 9.34$ & $84.88 \pm 4.01$ & $89.30 \pm 8.97$ & 0.855 & 0.555 & 0.948 \\
\hline Tetramer & $1.38 \pm 0.04$ & $18.35 \pm 2.56$ & $40.65 \pm 9.54$ & 0.474 & 0.019 & 0.319 \\
\hline Trimer & $7.83 \pm 1.29$ & $50.62 \pm 8.10$ & $63.57 \pm 12.30$ & 0.143 & 0.020 & 0.811 \\
\hline Dimer & $82.91 \pm 4.51$ & $88.97 \pm 6.43$ & $90.02 \pm 5.39$ & 0.4999 & 0.256 & 0.979 \\
\hline
\end{tabular}

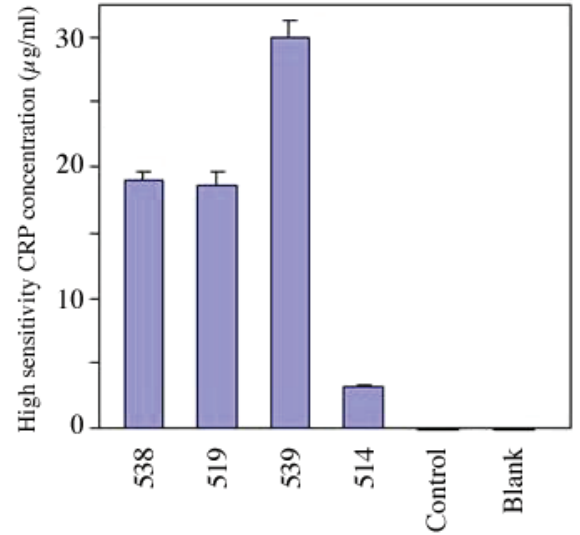

Figure 2. Concentrations of human high-sensitivity CRP in the blood of transgenic rat lines, as assessed by ELISA. 538, 519, 529 and 514 are 4 transgenic rat lines. Control, wild-type Sprague Dawley rats; blank, water control. Data are expressed as the mean \pm standard deviation. CRP, C-reactive protein.

and individual $\mathrm{c}$ was a patient with congenital heart disease as control. A hsCRP ELISA was performed prior to western blotting analysis. The CRP levels in the blood were $32.2 \mu \mathrm{g} / \mathrm{ml}$ in patient a, $25.8 \mu \mathrm{g} / \mathrm{ml}$ in patient $\mathrm{b}$ and undetectable in individual c. Non-reducing western blotting was performed using the M86284M monoclonal antibody (Meridian Life Sciences, Inc.). $\mathrm{CRP}$ was detected in pentamer, tetramer and dimer isoforms (Fig. 4B).

\section{Discussion}

The present study demonstrated that CRP may be present in various conformational isoforms in addition to pentamers.
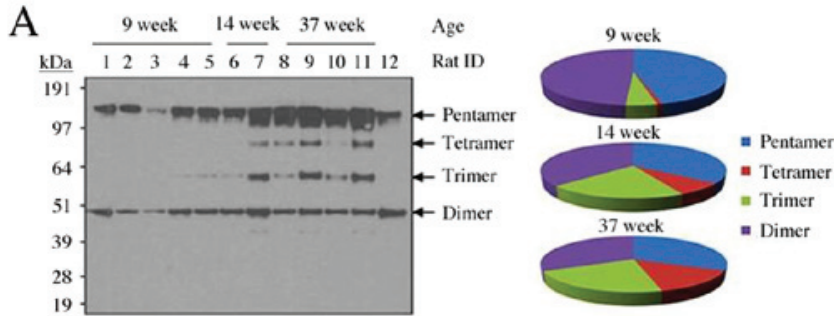

B

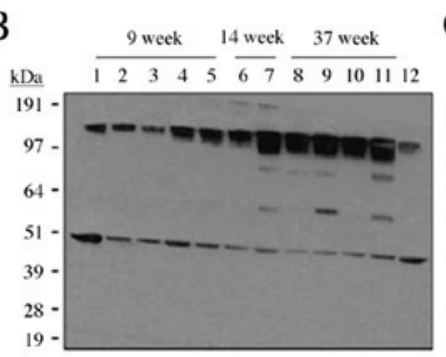

C 9week 37 week Age

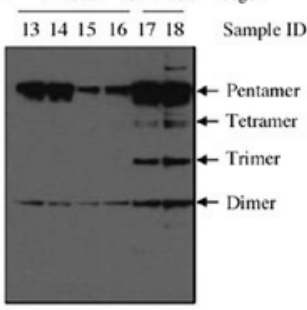

Figure 3. Detection of multimeric isoforms of CRP in the serum of transgenic rats at various ages, using 3 different anti-human CRP antibodies. (A) Detection of human CRP isoforms by non-reducing western blotting with anti-human CRP monoclonal antibody, catalog no. M86284M (Meridian Life Sciences, Inc.), and fractional composition of CRP multimeric isoforms for each age group following densitometric analysis. (B) The membrane was then stripped and re-blotted with anti-human CRP antibody, catalog no. ab52687 (Abcam). A total of 12 rats were analyzed, 11 transgenic rats from 4 transgenic lines and one wild-type control littermate (rat 12) at 38 weeks old. Rats 1, 2, 5 and 11 belong to transgenic line 519; rats $3,4,6,7$ and 10 belong to line 539; rat 8 belongs to line 538; rat 9 belongs to line 514. (C) Detection of human CRP isoforms by non-reducing western blotting with anti-human CRP antibody, catalog no. M86005M (Meridian Life Sciences, Inc), in the plasma (samples 13, 15 and 17) and serum (samples 14, 16 and 18) of three transgenic rats (line 519, F1 offspring). Samples 13, 14, 15 and 16 were from two 9-week old transgenic rats, whereas samples 17 and 18 were from a 37-week old transgenic rat. CRP, C-reactive protein. 

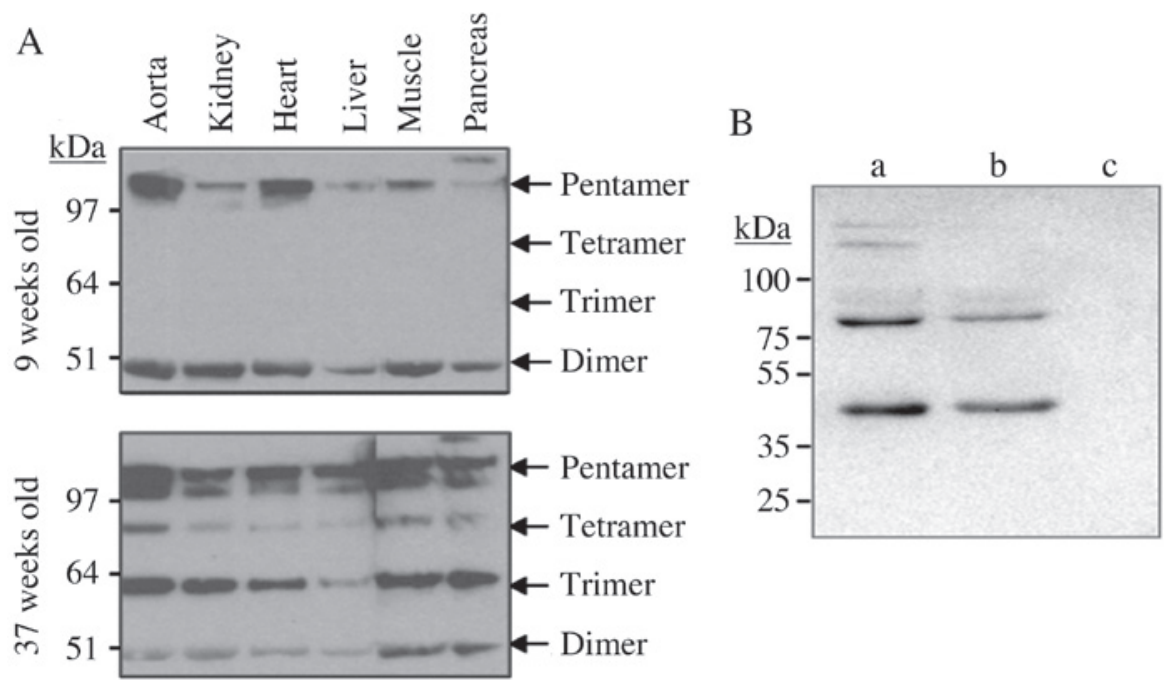

Figure 4. Multimeric isoforms of CRP in various tissues of transgenic rats and in human blood. (A) Human CRP isoforms were analyzed in the tissues of transgenic rats by non-reducing western blotting with an anti-human CRP monoclonal antibody (M86284M, Meridian Life Sciences, Inc.). The tissues were perfused to eliminate blood prior to processing. As for blood, dimer, trimer, tetramer and pentamer isoforms were detected in all six tissues, with the trimer and tetramer isoforms appearing only in the 37-week-old rats. (B) Three human subjects were recruited, among which individuals a and b were patients with coronary heart disease and individual c was a male with congenital heart disease who was recruited as a control. Non-reducing western blotting was performed using the M86284M monoclonal antibody (Meridian Life Sciences, Inc.). CRP was detected in pentamer, tetramer and dimer isoforms in patients a and b, and was undetectable in individual c. CRP, C-reactive protein.

As the conformation may be critically associated with the functionality of CRP $(12,15,16,24,25)$ the presence of these isoforms in various tissues indicted that CRP conformation should be considered when analyzing the pathological roles of CRP in cardiovascular disease and when using hsCRP as a marker for clinical diagnosis.

There are various possibilities that may result in the differences in the detection of CRP trimers and tetramers between young and older rats. First, the detection of additional bands in older rats may be due to a greater concentration of CRP, which may enable the detection of other isoforms besides the pentamer. Second, older rats may have certain physiological conditions that alter the aggregation of mCRP or the degradation of pCRP. For example, calcium and sodium concentration may be involved in CRP aggregation (8,26-30), and has been associated with diseases of the elderly. It is estimated that almost $60 \%$ of dietary calcium is absorbed during childhood and early adulthood; beyond 35 years old, the absorption rate typically decreases to $20 \%$. The possibility that pCRP dissociated in non-reducing gels during the western blotting procedure performed in the present study has been eliminated by using extreme denaturing conditions in denaturing gel western blotting, in which trimers were still observed (data not shown). The local calcium concentration in the gel during electrophoresis is unknown. However, even if calcium dictates the formation of the novel multimeric isoforms of CRP, as calcium levels vary in human patients and among different tissues, the discovery of other CPR isoforms may be important. The mechanisms underlying the age-associated appearance of CRP trimers and tetramers remain to be elucidated.

Conformation of CRP may be critically associated with its function. Previous studies have demonstrated that mCRP and pCRP have opposing functions: mCRP induced interleukin- 8 secretion by neutrophils (24) and human coronary artery endothelial cells (16), increased neutrophil-endothelial cell adhesion (25) and inhibited neutrophil apoptosis (31). By contrast, native pentameric CRP failed to affect neutrophil apoptosis (31). The accumulation of mCRP but not pCRP has been reported in human atherosclerosis (12). Therefore, mCRP may be the active isoform that contributes to atherogenesis by modulating monocyte behavior (32). The functional roles of trimeric and tetrameric CRP in different tissues remain unknown. It has been recently reported that CRP may be present in isoforms greater than pentamers (33). Taken together, these results suggest that CRP may exist in multiple conformational isoforms beyond the well-defined pentamers and monomers.

The association between increased concentrations of hsCRP and future cardiovascular events has been well established. The current ELISA-based hsCRP assays distinguish pentameric molecules from other multimeric CRP isoforms; furthermore, these serum-based ELISA assays do not provide information on CRP concentration and conformation in other tissues. At the transcriptional and protein levels, CRP is expressed at varying levels in different human tissues (13,34-36). The locally-produced CRP within tissues may exert its functions locally or systemically via the bloodstream. The recently identified CRP promoter mutation in cancers with enhanced CRP expression may support the functional importance of CRP in regulating local inflammation (37). It may be useful to determine whether the CRP levels and conformation within tissues is important in the pathogenesis of diseases; however, it is unclear if a polymorphism exists regarding CRP conformation in humans, and if the CRP conformational alterations are associated with disease pathogenesis and diagnosis.

In conclusion, the results of the present study suggested that CRP may exist in multiple multimeric isoforms. These results indicated that it may be beneficial to investigate CRP 
conformation, in addition to the CRP concentration currently measured as hsCRP in the clinic.

\section{References}

1. Nakou ES, Liberopoulos EN, Milionis HJ and Elisaf MS: The role of C-reactive protein in atherosclerotic cardiovascular disease: An overview. Curr Vasc Pharmacol 6: 258-270, 2008.

2. Bansal T, Pandey A, Deepa D and Asthana AK: C-reactive proten (CRP) and its association with periodontal disease: A brief review. J Clin Diagn Res 8: ZE21-ZE24, 2014.

3. Kones R: Rosuvastatin, inflammation, C-reactive protein, JUPITER, and primary prevention of cardiovascular disease-a perspective. Drug Des Devel Ther 4: 383-413, 2010.

4. Yeh ET and Willerson JT: Coming of age of C-reactive protein: Using inflammation markers in cardiology. Circulation 107 370-371, 2003.

5. Li Q, Kang T, Tian X, Ma Y, Li M, Richards J, Bythwood T, Wang Y, Li X, Liu D, et al: Multimeric stability of human C-reactive protein in archived specimens. PLoS One 8: e58094, 2013.

6. Kilpatrick JM and Volanakis JE: Molecular genetics, structure, and function of C-reactive protein. Immunol Res 10: 43-53, 1991

7. Bodman-Smith KB, Melendez AJ, Campbell I, Harrison PT, Allen JM and Raynes JG: C-reactive protein-mediated phagocytosis and phospholipase D signalling through the high-affinity receptor for immunoglobulin G (FcgammaRI). Immunology 107 : 252-260, 2002

8. Okemefuna AI, Nan R, Miller A, Gor J and Perkins SJ: Complement factor $\mathrm{H}$ binds at two independent sites to $\mathrm{C}$-reactive protein in acute phase concentrations. J Biol Chem 285 $1053-1065,2010$

9. Peisajovich A, Marnell L, Mold C and Du Clos TW: C-reactive protein at the interface between innate immunity and inflammation. Expert Rev Clin Immunol 4: 379-390, 2008.

10. Diehl EE, Haines GK III, Radosevich JA and Potempa LA: Immunohistochemical localization of modified C-reactive protein antigen in normal vascular tissue. Am J Med Sci 319: 79-83, 2000

11. Molins B, Peña E, Vilahur G, Mendieta C, Slevin M and Badimon L: C-reactive protein isoforms differ in their effects on thrombus growth. Arterioscler Thromb Vasc Biol 28: 2239-2246, 2008.

12. Eisenhardt SU, Habersberger J, Murphy A, Chen YC, Woollard KJ, Bassler N, Qian H, von Zur Muhlen C, Hagemeyer CE, Ahrens I, et al: Dissociation of pentameric to monomeric C-reactive protein on activated platelets localizes inflammation to atherosclerotic plaques. Circ Res 105: 128-137, 2009.

13. Ji SR, Ma L, Bai CJ, Shi JM, Li HY, Potempa LA, Filep JG, Zhao J and Wu Y: Monomeric C-reactive protein activates endothelial cells via interaction with lipid raft microdomains. FASEB J 23: 1806-1816, 2009

14. Mihlan M, Blom AM, Kupreishvili K, Lauer N, Stelzner K, Bergström F, Niessen HW and Zipfel PF: Monomeric C-reactive protein modulates classic complement activation on necrotic cells. FASEB J 25: 4198-4210, 2011.

15. Khreiss T, József L, Potempa LA and Filep JG: Opposing effects of C-reactive protein isoforms on shear-induced neutrophil-platelet adhesion and neutrophil aggregation in whole blood. Circulation 110: 2713-2720, 2004.

16. Khreiss T, József L, Potempa LA and Filep JG: Conformational rearrangement in C-reactive protein is required for proinflammatory actions on human endothelial cells. Circulation 109 : 2016-2022, 2004

17. Potempa LA, Zeller JM, Fiedel BA, Kinoshita CM and Gewurz H: Stimulation of human neutrophils, monocytes, and platelets by modified C-reactive protein (CRP) expressing a neoantigenic specificity. Inflammation 12: 391-405, 1988.

18. Schwedler SB, Amann K, Wernicke K, Krebs A, Nauck M, Wanner C, Potempa LA and Galle J: Native C-reactive protein increases whereas modified C-reactive protein reduces atherosclerosis in apolipoprotein E-knockout mice. Circulation 112: 1016-1023, 2005
19. Li Q, Ma Y, Li W, Xu W, Ma L, Fu G, Tian X, Wang Y, Li X, Bythwood T, et al: A promoter that drives gene expression preferentially in male transgenic rats. Transgenic Res 23: 341-349, 2014.

20. Li Q, Xu W, Cui Y, Ma L, Richards J, Li W, Ma Y, Fu G, Bythwood T, Wang Y, et al: A prelimininary exploration on DNA methylation of transgene across generations in transgenic rats. Sci Rep 5: 8292, 2015

21. Baltz ML, de Beer FC, Feinstein A, Munn EA, Milstein CP, Fletcher TC, March JF, Taylor J, Bruton C, Clamp JR, et al: Phylogenetic aspects of $\mathrm{C}$-reactive protein and related proteins. Ann N Y Acad Sci 389: 49-75, 1982.

22. Hammond DJ Jr, Singh SK, Thompson JA, Beeler BW, Rusiñol AE, Pangburn MK, Potempa LA and Agrawal A: Identification of acidic $\mathrm{pH}$-dependent ligands of pentameric C-reactive protein. J Biol Chem 285: 36235-36244, 2010.

23. Wang MY, Ji SR, Bai CJ, El Kebir D, Li HY, Shi JM, Zhu W, Costantino S, Zhou HH, Potempa LA, et al: A redox switch in C-reactive protein modulates activation of endothelial cells FASEB J 25: 3186-3196, 2011.

24. Khreiss T, József L, Potempa LA and Filep JG: Loss of pentameric symmetry in C-reactive protein induces interleukin-8 secretion through peroxynitrite signaling in human neutrophils. Circ Res 97: 690-697, 2005.

25. Zouki C, Haas B, Chan JS, Potempa LA and Filep JG: Loss of pentameric symmetry of C-reactive protein is associated with promotion of neutrophil-endothelial cell adhesion. J Immunol 167: 5355-5361, 2001

26. Motie M, Brockmeier S and Potempa LA: Binding of model soluble immune complexes to modified C-reactive protein. J Immunol 156: 4435-4441, 1996.

27. Wu Y, Ji SR, Wang HW and Sui SF: Study of the spontaneous dissociation of rabbit C-reactive protein. Biochemistry (Mosc) 67: 1377-1382, 2002

28. Ji SR, Wu Y, Zhu L, Potempa LA, Sheng FL, Lu W and Zhao J: Cell membranes and liposomes dissociate C-reactive protein (CRP) to form a new, biologically active structural intermediate: $\mathrm{mCRP}(\mathrm{m})$. FASEB J 21: 284-294, 2007.

29. Kresl JJ, Potempa LA and Anderson BE: Conversion of native oligomeric to a modified monomeric form of human $\mathrm{C}$-reactive protein. Int J Biochem Cell Biol 30: 1415-1426, 1998.

30. Okemefuna AI, Stach L, Rana S, Buetas AJ, Gor J and Perkins SJ: C-reactive protein exists in an $\mathrm{NaCl}$ concentration-dependent pentamer-decamer equilibrium in physiological buffer. J Biol Chem 285: 1041-1052, 2010.

31. Khreiss T, József L, Hossain S, Chan JS, Potempa LA and Filep JG: Loss of pentameric symmetry of C-reactive protein is associated with delayed apoptosis of human neutrophils. J Biol Chem 277: 40775-40781, 2002.

32. Zhao J and Shi XH: Study of the interaction of the C-reactive protein monomer with the U937 monocyte. Cell Mol Biol Lett 15: 485-495, 2010.

33. Asztalos BF, Horan MS, Horvath KV, McDermott AY, Chalasani NP and Schaefer EJ: Obesity associated molecular forms of C-reactive protein in human. PLoS One 9: e109238, 2014.

34. Beri A, Contractor T, Khasnis A and Thakur R: Statins and the reduction of sudden cardiac death: Antiarrhythmic or anti-ischemic effect? Am J Cardiovasc Drugs 10: 155-164, 2010.

35. Juma WM, Lira A, Marzuk A, Marzuk Z, Hakim AM and Thompson CS: C-reactive protein expression in a rodent model of chronic cerebral hypoperfusion. Brain Res 1414: 85-93, 2011.

36. Peyrin-Biroulet L, Gonzalez F, Dubuquoy L, Rousseaux C, Dubuquoy C, Decourcelle C, Saudemont A, Tachon M, Béclin E, Odou MF, et al: Mesenteric fat as a source of C reactive protein and as a target for bacterial translocation in Crohn's disease. Gut 61: 78-85, 2012.

37. Wang MY, Zhou HH, Zhang SC, Hui F, Zhu W, Su HX, Guo HY, Li XW, Ji SR and Wu Y: Recurrent mutations at C-reactive protein gene promoter SNP position -286 in human cancers. Cell Res 24: 505-508, 2014. 